\title{
Heike C. Opitz Der juristische und gesellschaftliche Umgang mit den Ansprüchen auf Teilzeitarbeit in den Niederlanden und in Deutschland
}

\section{A. Einleitung}

Wie gesellschaftliche Probleme juristisch zu lösen versucht werden, sagt viel über eine Gesellschaft aus. Dies wird auch an der Diskussion um den Teilzeitanspruch und den Anspruch auf Verlängerung der Arbeitszeit deutlich. Bei der Schaffung des Teilzeitanspruchs in $\$ 8 \mathrm{TzBfG}$ hat sich der deutsche Gesetzgeber an der niederländischen Regelung im Gesetz zur Anpassung der Arbeitszeit (Wet aanpassing arbeitsduur Waa) orientiert. ${ }^{I}$ Die deutsche Diskussion zeichnet sich jedoch im Vergleich mit den Niederlanden durch eine ideologische Debatte aus, die sowohl einen Teil der Probleme mit dem Anspruch als auch die positiven Ansätze nicht erkennt. Dagegen lässt sich in den Niederlanden gerade auch an dem Anspruch auf Anpassung der Arbeitszeit ein deutlich pragmatischerer Umgang mit Recht und flexiblere Lösungsversuche gesellschaftlicher Probleme feststellen.

Außer den Niederlanden haben zwar noch weitere Länder ausgeweitete Teilzeitmöglichkeiten. Auch in Belgien besteht ein Anspruch auf eine befristete Reduzierung der Arbeitszeit. ${ }^{2}$ Die Teilzeitquote lag im Jahr 200 I bei I 8,2\%. ${ }^{3}$ In Schweden existieren beim Elternurlaub bezahlte Möglichkeiten, die Arbeitszeit zu reduzieren. ${ }^{4}$ Die Teilzeitquote der Beschäftigten lag im Jahr $200 \mathrm{I}$ bei 24, I \%.5 Das Vereinigte Königreich weist im Jahr 200 I eine Teilzeitquote der Beschäftigten von $24,9 \%$ auf ${ }^{6}$ und hat neben den Niederlanden die höchste Teilzeitquote, allerdings hauptsächlich bei geringfügig Beschäftigten. ${ }^{7}$

Jedoch sind die Niederlande - das Land des »Flexicurity« - unbestritten Vorreiter hinsichtlich Teilzeitarbeit. Sie haben europaweit die höchste Teilzeitquote von $42 \%$ aller Beschäftigten (200I). ${ }^{8}$ Dort besteht Teilzeitarbeit nicht nur im Sektor der

\footnotetext{
I Dies wird sowohl am Gesetzgebungsverfahren als auch an der Ausgestaltung der Gesetze deutlich.

2 Siehe für einen Überblick über die Regelungen: http:/meta.fgov.be/pk/pkh/nlkh22.htm; der Rechtsanspruch wurde auf Grundlage der Tarifverträge Nr. 77, 77bis, 77ter geschaffen. Regelungen sind im Herstelwet van 22 januari 1985 , hoofstuk IV, afdeling $V$ zu finden. Die Regelungen zu der Finanzierung der Laufbahnunterbrechung sind im Gesetz Wet betreffende de verzoening van werkgelegenheid en kwaliteit van het leven (Gesetz zur Vereinbarung von Beschäftigung und Lebensqualität), belgisch staatsblad - I 5. 09. 200 I, 30949 ff. und belgisch staatsblad - I8. I2.200I, $43639 \mathrm{ff}$. geregelt worden und unterscheiden sich stark von den niederländischen und deutschen Ansprüchen auf Anpassung der Arbeitszeit; daneben bestehen auch Möglichkeiten, im Rahmen der Kindererziehung oder Pflege von Angehörigen eine Reduzierung der Arbeitszeit zu erreichen; Hinweise auf den belgischen Anspruch in: AuR Info, AuR 2002, I4 I f.

3 Europäische Kommission, Beschäftigung in Europa 2002, S. 174.

4 Köhler, EuroAS 2001, 2 17, 2 I 9.

5 Europäische Kommission (Fn. 3), S. I 87.

6 Europäische Kommission, ebd., S. 188.

7 Fagan/O’Reilly/Rubery, WSI Mitteilungen I999, 58, 62.

8 Europäische Kommission (Fn. 3), S. I 83 .
} 
geringfügig Beschäftigten, sondern auch bei höher qualifizierten und existenzsichern-

den Tätigkeiten. ${ }^{9}$ Bei Arbeitnehmerinnen kann sie als Standardarbeitszeitmodell bezeichnet werden, 71\% der Frauen arbeiten Teilzeit. Aber auch der Anteil der männlichen Arbeitnehmer liegt mit 20\% deutlich über dem Anteil der anderen europäischen Länder. ${ }^{10}$

Die Frage nach flexibleren Gestaltungen der Erwerbsbeziehungen zur Ermöglichung der Wahrnehmung von gesellschaftlichen Aufgaben, in den Niederlanden schon länger unter dem Stichwort Levensloopbaanbeleid diskutiert, wird auch in Deutschland immer wichtiger und gilt als eine Voraussetzung, um die Arbeitswelt den sich langfristig verändernden Umständen auf dem Arbeitsmarkt und in der Gesellschaft anzupassen.

In Deutschland arbeiten zurzeit nur $20 \%$ der Beschäftigten Teilzeit. Der Anteil ist auch hier bei den Frauen mit 39\% deutlich höher als der Anteil von 5\% bei den Männern. ${ }^{\text {I }}$ Auch hier ist Teilzeitarbeit ein Frauenphänomen. Möglicherweise könnten hier aber die Regelungen des TzBfG in Deutschland die Bedeutung von Teilzeitarbeit und dem Wechsel zwischen verschiedenen Arbeitszeiten verändern.

\section{B. Gesellschaftliche und rechtliche Entwicklung der Teilzeitarbeit}

In den Niederlanden wird seit über zehn Jahren in der rechtswissenschaftlichen Literatur $^{12}$ und im Parlament diskutiert, ob ein Recht auf Teilzeitarbeit eingeführt werden soll. ${ }^{13}$ Auch vor der Schaffung des Waa wurde in den Niederlanden insbesondere von Arbeitnehmern versucht, durch Klagen eine Verringerung der vertraglich festgelegten Arbeitszeit zu erreichen. Dies wurde auf dem Weg einer Teilkündigung oder nach dem Grundsatz der guten Arbeitgeberschaft beansprucht. Ein Recht auf Teilzeitarbeit wurde wegen einer fehlenden ausdrücklichen rechtlichen Regelung von den meisten Gerichten abgelehnt. ${ }^{14}$ Die Einführung des Rechtsanspruchs auf Anpassung der Arbeitszeit im Wet aanpassing arbeidsduur (Gesetz zur Anpassung der Arbeitszeit, Waa) am I. 7. 2000 wurde als Abschluss einer zehnjährigen Entwicklung angesehen.

Das deutsche TzBfG hingegen beruht auf keiner langjährigen Diskussion in der Rechtswissenschaft und Politik, sondern wurde innerhalb eines halben Jahres im Rahmen der Umsetzung der EG-Richtlinien RL 97/8I/EG und RL 99/70/ EG eingeführt. Dabei nahm sich der deutsche Gesetzgeber das Waa als Vorbild. Ziel war neben der Gleichstellung von Männern und Frauen und der besseren Vereinbarkeit von Beruf und Familie insbesondere die Behebung der hohen Arbeitslosigkeit. Die Reaktionen waren sehr viel negativer als in den Niederlanden. Die Arbeitgeberverbände lehnten das Gesetz vollständig ab. ${ }^{\text {is }}$ Neben dem Vorwurf der Überregulierung und des unzulässigen Eingriffs in die unternehmerische Entscheidungsfreiheit der Arbeitgeber wurde das Gesetz auch für kontraproduktiv gehalten und als Beschäftigungshindernis sowie als Gefährdung für den Stand-

9 Fagan/O’Reilly/Rubery (Fn. 7), 58, 62.

Io Europäische Kommission (Fn. 3), S. I 83 .

I I Europäische Kommission, ebd., S. I76.

I 2 Siehe hierzu: Olbers, SR I992, Nr. I 2, 332 ff.; Asscher-Vonk, SMA I993, 454 ff.; Burri, SMA I 993, 463 ff.

I 3 Beschreibung der verschiedenen Gesetzesinitiativen bei: Burri, Tijd delen - Deeltijd, gelijkheid en gender in Europees, Utrecht 2000, S. $589 \mathrm{ff}$.

I4 Untersuchung über die Rechtsprechung vor dem Inkrafttreten des Waa bei: Van Beek/van DoorneHuiskes/Veldman, Waa voortgangsrapportage, S. $43 \mathrm{ff}$.

Is Siehe: Süddeutsche Zeitung vom 22. I 2. 2000, S. 6. 
ort Deutschland betrachtet. ${ }^{16}$ Die Gewerkschaften begrüßten hingegen das Gesetz. ${ }^{17}$

Die Möglichkeiten, die Arbeitszeit zu verlängern, wurden in beiden Ländern weniger als die Teilzeitmöglichkeiten betrachtet. Bemerkenswert dabei ist insbesondere, dass in Deutschland eine vergleichbare Diskussion wie um den Teilzeitanspruch um den in \9 TzBfG normierten Rechtsanspruch auf Verlängerung der Arbeitszeit und die Verteilung der Arbeitsstunden nicht statt fand.

Auch nach vier Jahren Geltung werden die Wirkungen in den beiden Ländern sehr unterschiedlich beschrieben. In den Niederlanden wurde bei einer vom Gesetzgeber geforderten Zwischenbilanz des Waa festgestellt, dass den meisten Arbeitgebern das Waa bekannt sei, allerdings in kleinen Betrieben nicht die genauen Anforderungen des Gesetzes. Mit den Wünschen von Arbeitnehmern nach Anpassungen der Arbeitszeit werde pragmatisch umgegangen und oft auch entsprochen, um qualifiziertes Personal wegen des engen Arbeitsmarktes zu werben oder zu behalten. ${ }^{18}$

In Deutschland wurde bei zwei Untersuchungen des Instituts für Arbeitsmarkt- und Berufsforschung der Bundesanstalt für Arbeit (IAB) festgestellt, dass der Teilzeitanspruch im Jahr $200 \mathrm{I}$ von 85.000 Beschäftigten und im Jahr 2003 von I 27.000 Beschäftigten geltend gemacht wurde. Die Mehrzahl der Anträge wurden von Frauen gestellt (200I: 78\%; 2003: 73\%). Anträge auf Verlängerung der Arbeitszeit gab es 200 I so gut wie keine, mittlerweile kommt auf zwei Verringerungs- ein Verlängerungsantrag. In den meisten Fällen wurde diesen Anträgen, insbesondere in großen Betrieben, entsprochen. Die Verkürzung der Arbeitszeit wurde jeweils zur Hälfte durch produktionswirksame Maßnahmen oder beschäftigungswirksame Maßnahmen umgesetzt. Während das IAB daraus schlussfolgerte, dass das TzBfG reibungsarm, einvernehmlich und partnerschaftlich umgesetzt wurde, ${ }^{19}$ zeichnete die Deutsche Industrie und Handelskammer (DIHK) im Jahr 200I ein gänzlich anderes Bild auf Grundlage einer Befragung ihrer Mitglieder. Hiernach sei das TzBfG kontraproduktiv. Schon bei der Einstellungspraxis übten die Unternehmen Zurückhaltung, wenn sie ein Teilzeitinteresse befürchteten. Der Rechtsanspruch müsse abgeschafft werden. ${ }^{20}$

Eine Evaluation der Wirkungen des TzBfG fehlt bisher. Zwar gab das Bundesministerium für Arbeit und Sozialordnung eine solche bei dem Institut zur Erforschung sozialer Chancen (ISO) und ifo Institut für Wirtschaftsforschung für den Zeitraum September $200 \mathrm{I}$ bis 2003 in Auftrag, eine Veröffentlichung der Ergebnisse fehlt aber bis jetzt. Dabei wäre zur Versachlichung der Debatte eine Evaluation hilfreich. In den Niederlanden ist bereits eine erste Evaluation erfolgt. ${ }^{21}$

Im Gegensatz zu den Niederlanden gibt es weiterhin Bemühungen, das TzBfG zu verändern bzw. abzuschaffen. So brachte die CDU/CSU-Fraktion im Juni 2003 einen neuen Gesetzesentwurf ein, um das TzBfG zu ändern. Ziel war insbesondere, Teilzeitarbeit nur aus familiären Gründen zu ermöglichen. ${ }^{22}$ Auch der Deutsche Juristentag forderte im September 2004 im Rahmen einer skurrilen Abstimmung die Abschaffung der Ansprüche. ${ }^{23}$

I6 Siehe Stellungnahme der Bundesvereinigung der Deutschen Arbeitgeberverbände (BDA) bei der öffentlichen Anhörung von Sachverständigen, BT-Drs. I 4/4625, S. I7.

I7 Siehe Stellungnahme des Deutschen Gewerkschaftsbundes (DGB) bei der öffentlichen Anhörung von Sachverständigen, BT-Drs. I4/4625, S. I7.

I 8 Van Beek/van Doorne-Huiskes/Veldman (Fn. I4), S. XIIf.

I 9 Magvas/Spitznagel, IAB-Kurzbericht Nr. 23/2002; Wanger, IAB-Kurzbericht Nr. I 8/2004.

20 DIHK, Mehr Konflikte, weniger Flexibilität - Erfahrungen mit dem TzBfG, S. ı०.

2 I Ergebnisse siehe: Van Beek/van Doorne-Huiskes/Veldman (Fn. I4).

22 BT-Drs. I $/$ I I 82.

23 Siehe Berichterstattung in der FAZ vom 24. 9. 2004 , S. 6. 
Grundlegende gesellschaftliche und rechtliche Rahmenbedingungen sind in den Niederlanden und in Deutschland ähnlich. In beiden Ländern entwickelte sich die Diskussion über die Anpassung der Arbeitszeit zunächst unter dem Aspekt der Teilzeitarbeit. So wurden in beiden Ländern für Teilzeitarbeitnehmer auf Grundlage von europäischen Richtlinien zunächst Antidiskriminierungsmaßnahmen geschaffen. ${ }^{24}$ In den Niederlanden wurde Teilzeitarbeit seit Beginn der 8oer Jahre gefördert, zunächst nur, um eine hohe Arbeitslosigkeit zu bekämpfen. Sie diente schließlich auch einer wachsenden Partizipation von Frauen auf dem Arbeitsmarkt und wird heute vor allem unter dem Aspekt der Vereinbarkeit von Beruf und Familie diskutiert. In Deutschland wurde ein ähnlicher Effekt für den Arbeitsmarkt in den 8oer Jahren über eine generelle Arbeitszeitverkürzung zu erreichen versucht. Die systematische Förderung von Teilzeitarbeit wurde erst ab den 9oer Jahren diskutiert.

Schon an dieser Entwicklung werden Unterschiede deutlich. Während sich in dem Akkord van Wassenaar (Vereinbarung von Wassenaar) schon 1982 alle gesellschaftlichen Akteure auf einen Kurs zur Bekämpfung der Arbeitslosigkeit verständigten, herrschte in Deutschland Konfrontation zwischen den Akteuren. Das niederländische Poldermodell, das stark auf Konsens ausgerichtet ist, hat sich in Deutschland, trotz Versuchen, ähnliche Formen einzuführen, nicht durchsetzen können.25

Beide Länder haben Möglichkeiten für eine Freistellung von der Erwerbstätigkeit nach der Geburt und in den ersten Lebensjahren eines Kindes geschaffen. Die rechtlichen Regelungen sind der Elternurlaub in den Niederlanden und die Elternzeit in Deutschland. In den Niederlanden, wo die Möglichkeit eines Elterurlaubs erst später als in Deutschland eingeführt wurde, ist die zeitliche Dauer deutlich kürzer als in Deutschland. Sie beträgt I 3 Wochen, kann allerdings als Teilzeit-Elternurlaub genommen und entsprechend ausgeweitet werden (Art. 6:1-6:9 Gesetz Arbeit und Sorge [nachfolgend abgekürzt WA\&Z]). Die Teilzeitmöglichkeiten kann der Arbeitgeber dabei nicht ablehnen. In Deutschland besteht hingegen ein Freistellungsanspruch bis zu 3 Jahren. Daneben hat der Arbeitnehmer einen Anspruch, in der Elternzeit Teilzeit bei seinem Arbeitgeber zu arbeiten. Allerdings kann der Arbeitgeber die Teilzeit in der Elternzeit aus dringenden betrieblichen Gründen ablehnen. ${ }^{26}$

Das niederländische und das deutsche Recht sind sehr ähnlich strukturiert. So weisen das Zivilrecht, das Arbeitsrecht und das Zivilprozessrecht strukturelle Gemeinsamkeiten auf. Die Anwendung in der Rechtspraxis verläuft allerdings sehr unterschiedlich. In beiden Ländern besteht eine andere Rechtskultur. Während in den Niederlanden eine wenig dogmatische und sehr informelle Anwendung des Rechts stattfindet, wird in Deutschland auch in der Praxis das Recht dogmatisch und formal angewendet. Beispiel hierfür sind das niederländische Rechtsinstitut der guten Arbeitgeber- und Arbeitnehmerschaft in Art.7:6I I BW, welches zwar mit der Fürsorgepflicht des Arbeitgebers und der Treuepflicht des Arbeitnehmers in Deutschland verglichen werden kann, jedoch einen so weiten Anwendungsbereich findet, dass auch feste Vertragsbestandteile des Arbeitsvertrags abänderbar werden. ${ }^{27}$

24 Siehe zur Entwicklung der so genannten atypischen Beschäftigungsverhältnisse in Deutschland: Schiek, Das Teilzeit- und Befristungsgesetz, KJ 2002, S. I 8 ff.

25 Schmid, Leviathan, Jhg. 25, Heft 3, I997, S. 303, 325.

$26 \mathrm{Burri} /$ Opitz/Veldman,Work-family Policies on Working Time put into Practice, IJCLLIR 2003, 32 I, $325 \mathrm{f}$.

27 Opitz, Die Rechtsansprüche auf Anpassung der Arbeitszeit, \$2, C., I., I., a). 
Ein Bespiel aus dem Prozessrecht ist die Möglichkeit des Eilverfahrens. Während in Deutschland eine einstweilige Verfügung Ausnahmecharakter hat, hat sich in den Niederlanden das Eilverfahren als Standardverfahren im Prozess durchgesetzt. ${ }^{28}$ Die beiden nationalen Gesetze sind in unterschiedliche juristische Kontexte eingegliedert worden. In den Niederlanden wird das Waa als Teil des WA\&Z eingegliedert. Das WA\&Z regelt Beurlaubungs- und Freistellungsmöglichkeiten im Rahmen familiärer Tätigkeiten. Das TzBfG regelt neben den Teilzeit- auch die Befristungsmöglichkeiten für ein Arbeitsverhältnis. Des Weiteren ist ein Diskriminierungsverbot für Teilzeit- und befristet Beschäftigte enthalten. In beiden Ländern gibt es neben diesen Ansprüchen noch spezielle Ansprüche in Gesetzen oder Tarifverträgen, die allerdings häufig an spezielle Gründe, meistens familiäre Tätigkeiten, anknüpfen.

\section{Die Rechtsansprüche auf Teilzeitarbeit und Verlängerung der Arbeitszeit}

Beim Vergleich der beiden Ansprüche auf Teilzeitarbeit und auf Verlängerung der Arbeitszeit wird das unterschiedliche Rechtsverständnis in den Niederlanden und in Deutschland deutlich. Zunächst weist die Entwicklung der Ansprüche große Unterschiede auf. Die Gesetze, die auf den ersten Blick sehr ähnlich erscheinen, sind in wesentlichen Punkten unterschiedlich. Schließlich verdeutlicht auch die Diskussion in der juristischen Literatur um die Rechtsansprüche und die konkrete Anwendung in der Rechtsprechung, dass große Differenzen bestehen.

\section{Die Struktur der Rechtsansprüche}

Schon an der Struktur der beiden Gesetze wird das unterschiedliche Verständnis von Teilzeitarbeit in den beiden Ländern deutlich. In den Niederlanden ist der Anspruch auf Anpassung der Arbeitszeit einheitlich als ein Anspruch geregelt, der grundsätzlich unter denselben Voraussetzungen Anwendung findet. Damit wird zwischen der Verringerung und Verlängerung der Arbeitszeit nicht unterschieden. Allerdings wird die Verteilung der Arbeitsstunden anders als die Anpassung der Arbeitszeit behandelt und kann von dem Arbeitgeber leichter abgelehnt werden.

Das deutsche Gesetz hingegen hat den Fokus auf den Teilzeitanspruch gelegt. Dieser ist sehr ähnlich wie der niederländische Anspruch auf Anpassung der Arbeitszeit. Die Verteilung der Arbeitsstunden wird grundsätzlich wie die Verringerung der Arbeitszeit behandelt. Der Anspruch auf Verlängerung weist weder mit der niederländischen noch mit der deutschen Regelung des Teilzeitanspruchs Ähnlichkeiten auf.

\section{Der Inbalt der Rechtsansprüche}

Die Voraussetzungen des Rechtsanspruchs auf Anpassung der Arbeitszeit sind in den Niederlanden in Art. 2 Waa und in Deutschland in $\$ 8$ TzBfG normiert. In beiden Ländern müssen sowohl Arbeitnehmer wie Arbeitgeber ein klares Verfahren bei der Beantragung der Teilzeitarbeit einhalten. Es gilt eine Wartefrist, bis zu der ein Arbeitnehmer das erste Mal einen Antrag stellen kann. Der Arbeitgeber muss in einer gewissen Frist reagieren, ansonsten findet eine automatische Anpassung der Arbeitszeit statt. Zudem finden die Regelungen erst bei einer bestimmten Unternehmensgröße Anwendung. ${ }^{29}$

28 Blankenberg/Bruinsma, Dutch Legal Culture, S. $39 \mathrm{ff}$.

29 Beschreibung der Voraussetzungen bei: Jakobs/Schmidt, IJCLLIR 200I, 37 I ff., Burri/Opitz/Veldman (Fn. 26), 32I, $328 \mathrm{ff}$. 
Das Waa schreibt vor, dass der Arbeitgeber einem Antrag des Arbeitnehmers zustimmen muss, es sei denn, dass schwerwiegende betriebliche Belange gegen den Antrag sprechen (Art. 2 Abs. 5 Waa). Eine Beschreibung dieser Ablehnungsgründe findet in Art. 2 Abs. 8 und 9 Waa statt. Danach liegt bei der Verringerung der Arbeitszeit in jedem Fall dann ein schwerwiegender betrieblicher Belang vor, wenn ernsthafte Probleme für die Betriebsführung bei der Wiederbesetzung der freiwerdenden Stunden, auf dem Gebiet der Sicherheit oder bei der Erstellung von Zeit- und Schichtplänen auftreten (Art. 2 Abs. 8 Waa). Bei der Verlängerung der Arbeitszeit liegt ein schwerwiegender betrieblicher Belang insbesondere vor, wenn ernsthafte Probleme auftreten, bei Belangen finanzieller und organisatorischer Art, dem Fehlen von ausreichenden Arbeitsmöglichkeiten oder unzureichendem festgelegten Raum im Stellenplan oder Personalbudget (Art. 2 Abs. 9 Waa). Diese Regelbeispiele stellen keine abschließende Aufzählung dar. Die Verteilung der Arbeitsstunden soll der Arbeitgeber grundsätzlich übereinstimmend mit den Wünschen des Arbeitnehmers festlegen. Allerdings kann er hiervon abweichen, wenn das betriebliche Interesse das Interesse des Arbeitnehmers an einer bestimmten Verteilung überwiegt (Art. 2 Abs. 6 Waa). Damit findet hinsichtlich der Verteilung der Arbeitsstunden nur eine Interessenabwägung statt.

In Deutschland muss der Arbeitgeber der Verringerung der Arbeitszeit zustimmen und die Verteilung der Arbeitszeit entsprechend den Wünschen des Arbeitnehmers festlegen, es sei denn, dass betriebliche Gründe dem entgegenstehen ( $\$ 8$ Abs. 4 Satz I TzBfG). Die betrieblichen Gründe sind in den Regelbeispielen des $\int 8$ Abs. 4 Satz 2 TzBfG weiter erläutert. Danach liegt ein betrieblicher Grund insbesondere vor, wenn die Verringerung der Arbeitszeit die Organisation, den Arbeitsablauf oder die Sicherheit des Betriebes wesentlich beeinträchtigt oder unverhältnismäßige Kosten verursacht. Im Gegensatz zu den Niederlanden wird die Verteilung der Arbeitszeit damit identisch mit der Verringerung der Arbeitszeit behandelt.

Der Rechtsanspruch auf Verlängerung der Arbeitszeit in $\$ 9$ TzBfG weist wenig Ähnlichkeit auf mit der niederländischen Regelung in Art. 2 Waa auf. Zunächst gibt es keine Einschränkung des Anwendungsbereichs, auch wird eine bestimmte Dauer der Betriebszugehörigkeit nicht vorausgesetzt. Voraussetzung ist, dass der Arbeitnehmer dem Arbeitgeber seinen Verlängerungswunsch seiner vertraglich vereinbarten Arbeitszeit anzeigt. Bei einem entsprechenden freien Arbeitsplatz ist der Arbeitnehmer bevorzugt zu berücksichtigen. Der Arbeitgeber kann den Verlängerungswunsch ablehnen, wenn dringende betriebliche Gründe oder die Arbeitszeitwünsche anderer Arbeitnehmer entgegenstehen. Eine Regelung zur Verteilung der Arbeitszeit fehlt, sodass das normale Direktionsrecht des Arbeitgebers gilt.

In beiden Ländern wurde mit dem Recht auf Anpassung der Arbeitszeit und der Verteilung der Arbeitsstunden kein isoliertes Recht auf eine bestimmte Verteilung der Arbeitsstunden und auch kein Recht auf befristete Anpassung der Arbeitszeit geschaffen. Mit der Reduzierung der Arbeitszeit reduziert sich entsprechend auch der Lohn des Arbeitnehmers.

\section{Generelle Kritik an den Ansprüchen}

Das Waa hat in der niederländischen rechtswissenschaftlichen Literatur generelle Kritik ausgelöst. Auf der einen Seite wurde das Gesetz als zu weitgehend aufgefasst und insbesondere die damit verbundene Einschränkung der Vertragsfreiheit der Arbeitgeber kritisiert. ${ }^{\circ}$ Diese Kritiker bewerteten das Waa als wenig einflussreich 
und meinten, dass es kaum Auswirkungen auf die Vertragsbeziehungen haben werde. $^{3 \mathrm{I}}$ Auf der anderen Seite wurde das Waa positiv als erster Schritt zu einer neuen Verteilung der bezahlten und unbezahlten Arbeit zwischen Männern und Frauen betrachtet und aus diesem Grunde für notwendig erachtet. ${ }^{32}$

Die Auslegung der einzelnen Regelungen des Waa erfolgte jedoch relativ einheitlich, und es lassen sich nur wenige Streitstände feststellen. ${ }^{33}$

In Deutschland wurde die Regelung zur Verringerung der Arbeitszeit vielfach als kontraproduktiv und verfehlt erachtet. Vereinzelt wurde bezweifelt, ob ein Teilzeitanspruch verfassungsgemäß sei und aus den Bedenken heraus eine Auslegung der betrieblichen Gründe gefordert, die dem Arbeitgeber möglichst großen Freiraum bei der Ablehnung lässt. ${ }^{34}$ In Betracht käme ein Verstoß gegen die Berufsfreiheit des Arbeitgebers in Art. I 2 Abs. I GG und gegen die Vertragsfreiheit in Art. 2 Abs. I GG. Allerdings können beide Grundrechte durch vernünftige Überlegungen des Gemeinwohls eingeschränkt werden, deren Bestimmung grundsätzlich dem Gesetzgeber obliegt.35 Diese liegen hier in dem Ziel des Gesetzgebers, die Arbeitslosigkeit zu bekämpfen und die Vereinbarkeit von Beruf und Familie zu verbessern. Daher liegt kein Verstoß gegen Verfassungsrecht vor. ${ }^{36}$ Die teilweise geforderte einschränkende Auslegung lässt sich somit nicht aus dem Grundgesetz herleiten. Aus dieser Kontroverse folgten in Deutschland insbesondere im Rahmen des Teilzeitanspruchs viele Auslegungsprobleme.

\section{Ablebnungsmöglichkeiten für den Arbeitgeber im Rabmen der Ansprüche}

Die entscheidende Frage in beiden Ländern ist, aus welchen Gründen Arbeitgeber einen Antrag auf Teilzeitarbeit ablehnen können.

\section{a) Bewertung in der niederländischen Literatur und Rechtsprechung}

Viele Stimmen in der niederländischen Literatur sahen die Ablehnungsmöglichkeiten bei der Anpassung der Arbeitszeit als wenig aussagekräftig an. Die Beispiele, die im Gesetzgebungsprozess genannt wurden, wurden für nicht hilfreich erachtet, da sie sich auf die Rechtsprechung vor Geltung des Waa bezogen. So werde es letztendlich den Gerichten überlassen, einheitliche Bewertungsmaßstäbe zu entwickeln. ${ }^{37}$

Der größte Unterschied gegenüber der Rechtslage vor Geltung des Waa sei, dass die Beschwerden des Arbeitgebers nun zu ernsthaften Problemen führen müssen. Dies führt im Vergleich zur Rechtsprechung vor Geltung des Waa zu einer Erschwerung der Beweislast des Arbeitgebers. Die Erfolgsaussichten der Klagen der Arbeitnehmer sind damit gestiegen. Des Weiteren findet durch die Nennung der ernsthaften Probleme in den Regelbeispielen eine Qualifizierung der Ablehnungsgründe statt, die alle Ablehnungsgründe erfüllen müssen. ${ }^{38}$

Die niederländischen Gerichte wenden einen einheitlichen Maßstab zur Überprüfung der schwerwiegenden betrieblichen Belange an. Die Gründe des Arbeitgebers sind

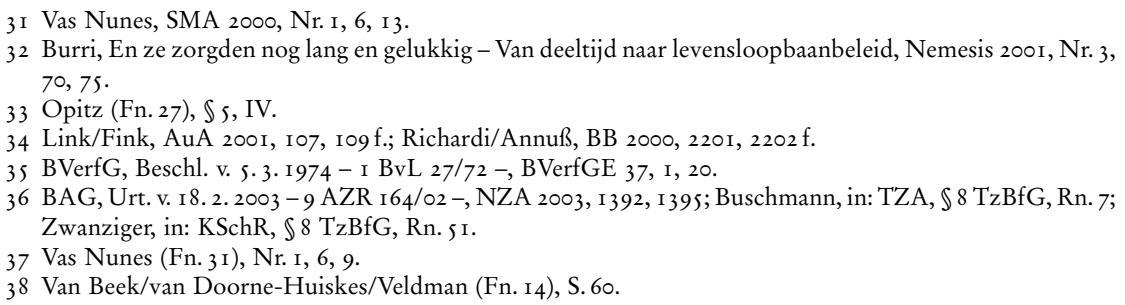


nach der Rechtsprechung vollständig überprüfbar (so genannte integrale toetsing). ${ }^{39}$

Des Weiteren forderten die Gerichte, dass die vorgetragenen schwerwiegenden betrieblichen Belange auch zu ernsthaften Problemen führen müssen..$^{\circ}$ Vielfach wird auf die Erfahrungen mit Teilzeitarbeit während des Elternurlaubs Bezug genommen . ${ }^{1 \mathrm{I}}$ Schwerwiegende betriebliche Belange des Arbeitgebers wurden daher nur selten von den Gerichten anerkannt, sodass in den meisten Fällen die gewünschte Reduzierung der Arbeitszeit erreicht wurde. ${ }^{42}$

\section{b) Bewertung in der deutschen Literatur und Rechtsprechung}

Während die schwerwiegenden betrieblichen Belange in den Niederlanden relativ einheitlich eingeschätzt wurden, sind in der deutschen rechtswissenschaftlichen Literatur und Rechtsprechung strittig, welche Anforderungen an die betrieblichen Gründe, die den Arbeitgeber zur Ablehnung berechtigen, zu stellen sind.

Einheitlich wurde festgestellt, dass den Arbeitgeber die Beweislast für das Vorliegen der betrieblichen Gründe trifft. Eine reine Behauptung ist nicht ausreichend, vielmehr muss der Arbeitgeber rationale und nachvollziehbare Gründe vortragen. An betriebliche Gründe sind weniger hohe Anforderungen als an dringende betriebliche Gründe zu stellen. ${ }^{43}$

\section{aa) Voraussetzung der wesentlichen Beeinträchtigung}

Fraglich ist, welche Bedeutung der in den Regelbeispielen geforderten wesentlichen Beeinträchtigung des Betriebes zukommt. Während dies teilweise als Redaktionsversehen gewertet und eine einschränkende Auslegung 44 bis hin zur vollständigen Nichtbeachtung gefordert wurde, ${ }^{45}$ betrachteten andere Stimmen dies als notwendige Qualifikation der betrieblichen Gründe. ${ }^{6}$ Trotz einiger systematischer Argumente ist dies überzeugend, da ansonsten das Ziel des TzBfG nicht erreicht werden könnte. Das Bundesarbeitsgericht hat mittlerweile entschieden, dass betriebliche Gründe nur vorliegen, wenn sie auch zu einer wesentlichen Beeinträchtigung des Betriebs führen. 47

\section{bb) Die unternehmerische Entscheidung als betrieblicher Grund}

Ein weiteres Problem stellte sich bei der Frage, ob eine unternehmerische Entscheidung ein betrieblicher Grund sein könne und inwieweit dieser durch die Gerichte überprüfbar ist. Anders als in den Niederlanden wird in Deutschland eine unternehme-

39 Gerechtshof Arnhem, I0. I2. 2002, www.rechtspraak.nl, LJN-Nr.: Af 2275, Zaaknr.: 02/565. Bei der Rechtsprechung vor der Geltung des Waa wurde nur eine marginale toetsing, also eine eingeschränkte Überprüfung der Gründe des Arbeitgebers vorgenommen.

40 Gerechtshof Arnhem, I0. I 2. 2002, ebd.

4I Gerechtshof Arnhem, IO. I 2. 2002, ebd.; Ktr. Amsterdam, 26. II. 2002, JAR 2002, 28 I; Rechtbank'sHertogenbosch, 7. I. 2003, TAR 2003, 64.

42 Arkel/Smits, SR 200I, Nr. I I, 295, 299; Veldman, Nemesis 2003, Nr. I, 28, 30.

43 BAG, Urt. v. I 8. 2. 2003 -9 AZR I64/02-, NZA 2003, I 392, I 394 f.; bestätigend BAG, Urt. v. I 9. 8. 2003-9 AZR 542/02 - AP TzBfG $\$ 8$ Nr. 4; BAG, Urt. v. I6. 3. 2004 - 9 AZR 323/03 -, Rn. 79, www.bundesarbeitsgericht.de.

44 Beckschulze, DB 200I, 2598; Rolfs, RdA 200I, I 29, I36.

45 Hromadka, NJW 2001, 400, 402.

46 Stein, AuR 2003, 99, I02 f., Däubler, ZIP 200I, 217, 219; Zwanziger, in: KSchG, $\$ 8$ TzBfG, Rn. 27.

47 BAG, Urt. v. I 8. 2. 2003 - 9 AZR I64/02 -, NZA 2003, I 392, I 395; bestätigend BAG, Urt. v. I 9. 8. 2003-9 AZR 542/02 -, AP TzBfG \8 Nr. 4; BAG, 30. 9. 2003-9 AZR 665/02 -, AP Nr. s zu \8 TzBfG; BAG, Urt. v. I6. 3. 2004 -9 AZR 323/03 -, Rn. 79 (Fn. 43); BAG, 27. 4. 2004-522/03-, NZA 2004, S. I 225 ff.; BAG, 20. 7. $2004-626 / 0_{3}-$, AP Nr. 9 zu $\$ 8$ TzBfG. 
rische Entscheidung nur eingeschränkt von den Gerichten überprüft. Allerdings kann daraus nicht abgeleitet werden, dass schon die Behauptung einer unternehmerischen Entscheidung zur Ablehnung des Teilzeitanspruches ausreicht. Dies würde allerdings zur Bedeutungslosigkeit des Gesetzes führen. Auch aus der grundgesetzlich geschützten Berufsfreiheit folgt nichts anderes, da auch diese aus vernünftigen Gründen des Gemeinwohls eingeschränkt werden kann. Die unternehmerische Entscheidung an sich, also ohne weitere Spezifizierung, kann keinen betrieblichen Grund darstellen. $4^{8}$

Durch die Entscheidungen des Bundesarbeitsgerichts ist mittlerweile geklärt, dass höhere Anforderungen zu stellen sind. Die unternehmerische Entscheidungsfreiheit führt nicht dazu, dass ein Organisationskonzept nicht überprüfbar sei. Der Arbeitgeber muss dessen tatsächliches Vorliegen und seine konsequente Durchführung beweisen. 49

\section{cc) Erfolgsaussichten der Klagen}

Dass viele Klagen von Arbeitnehmern in Deutschland erfolgreich waren, erklärt sich auch damit, dass Arbeitgeber oft unterließen, ihre vorgetragenen betrieblichen Gründe auch zu beweisen. ${ }^{\circ}$ Vielfach wurden nur Schlagworte gegen die gewünschte Teilzeitarbeit angeführt, ${ }^{1}$ was mehr auf Vorbehalte als auf reale Probleme mit der Teilzeitarbeit hinweist.

\section{Verteilung der Arbeitsstunden}

Deutlich anders ist die Situation bei der Verteilung der Arbeitsstunden. Während in den Niederlanden nur eine Interessenabwägung zwischen den Interessen des Arbeitgebers und des Arbeitnehmers vorgenommen wird, kann in Deutschland der Arbeitgeber nur aus betrieblichen Gründen die gewünschte Verteilung der Arbeitsstunden ablehnen.

In den Niederlanden wurde der Maßstab der Interessenabwägung bei der Verteilung der Arbeitszeit in der Literatur kritisiert, da das eigentliche Ziel des Waa, nämlich eine bessere Vereinbarkeit von Beruf und Familie, damit gefährdet würde. Gerade bei familiären Verpflichtungen seien Arbeitnehmer auch auf eine bestimmte Verteilung der Arbeitszeit angewiesen.52

Bei der Interessenabwägung gaben die Richter meistens dem Wunsch des Arbeitgebers statt. Für Arbeitnehmer ist so eine Anpassung der Arbeitszeit gut durchsetzbar, deutlich schwieriger ist es aber, auch die gewünschte Verteilung zu erlangen. Die Gerichte scheinen dies als Ausgleich für den Arbeitgeber zu begreifen, der schon eine Anpassung der Arbeitszeit akzeptieren musste. 53

Die Verteilung der Arbeitsstunden fand in Deutschland nur wenig Beachtung und wurde prinzipiell identisch wie die Verringerung der Arbeitszeit betrachtet. Ihre besondere Bedeutung für Arbeitgeber und Arbeitnehmer wurde nicht thematisiert.

48 Buschmann (Fn. 36), $\$ 8$ TzBfG, Rn. 32; Zwanziger (Fn. 36), \4 TzBfG, Rn. 22; 88 TzBfG, Rn. 27; Schmidt, AuR 2002, 245, 248; Däubler (Fn.46), 217, 219; a. A.: Meinel/Heyn/Herms, TzBfG, $\mathbb{8}$, Rn. 53; Mengel, in: Annuß/Thüsing, $\$ 8$, Rn. I29; Kliemt, NZA 2001, 63, 65 f.; Preis/Gotthardt, DB 2000, $2065,2068$.

49 BAG, Urt. v. I 8. 2. 2003 - 9 AZR I64/02 -, NZA 2003, I 392, I 395 ff.; bestätigend: BAG, Urt. v. I 9. 8. 20039 AZR 542/02 -, AP TzBfG \8 Nr. 4; BAG, 30. 9. 2003 -9 AZR 665/02 -, AP TzBfG $₫ 8$ Nr. 5; BAG, Urt. v. 16.3.2004 - 9 AZR 323/03 - (Fn. 43), Rn. 79; BAG, 27.4. 2004 - 522/03 -, NZA 2004, S. I 225 ; BAG, 20. 7. $2004-626 / 03-$, AP TzBfG $\$ 8$ Nr. 9 .

50 Schiek (Fn. 24), S. I 8, 4 I.

5I ArbG Stuttgart, Urt. v. 5.7.200I - 21 Ca 2762/0I -, NZA 200I, 968, 970.

52 Burri (Fn. 32), 70, 76.

53 Van Beek/van Doorne-Huiskes/Veldman (Fn. I4), S. 54 . 
Strittig war, inwieweit bei der Ablehnung einer gewünschten Verteilung der Arbeitsstunden auch eine wesentliche Beeinträchtigung erforderlich ist. ${ }^{54}$ Das Bundesarbeitsgericht hat nun entschieden, dass ein einheitlicher Maßstab anzuwenden sei und eine wesentliche Beeinträchtigung zur Ablehnung einer gewünschten Verteilung der Arbeitsstunden notwendig sei.ss

\section{Ablebnungsmöglichkeiten bei der Verlängerung der Arbeitszeit}

In Deutschland wurde der Rechtsanspruch auf Verlängerung der Arbeitszeit völlig anders als der Rechtsanspruch auf Verringerung der Arbeitszeit gestaltet. Eine vergleichbar breite und strittige Auseinandersetzung wie beim Teilzeitanspruch fand nicht statt. Dies ist umso erstaunlicher, da gerade die Verlängerung der Arbeitszeit deutlich stärkere finanzielle Folgen für den Arbeitgeber haben kann und ihm die Möglichkeit beschnitten wird, frei über die Besetzung der Arbeitsplätze zu entscheiden.

Zur Rechtsprechung zum Rechtsanspruch auf Verlängerung der Arbeitszeit lässt sich in beiden Ländern nur auf zwei Entscheidungen zurückgreifen, so dass allgemeine Schlussfolgerungen nicht möglich sind. ${ }^{6}$

Interessant an der einen niederländischen Entscheidung ist, dass festgestellt wurde, dass die unternehmerische Entscheidungsfreiheit hinsichtlich der Besetzung freier Stellen durch den Rechtsanspruch auf Verlängerung der Arbeitszeit im Waa eingeschränkt worden sei. ${ }^{57}$

In Deutschland existieren eine Entscheidung des Bundesarbeitsgerichts zu einer vergleichbaren Tarifnorm und eine Entscheidung eines Arbeitsgerichtes. Dabei stellt das Bundesarbeitsgericht fest, dass der Arbeitnehmer auch bei anderweitiger Besetzung des Arbeitsplatzes einen weiter bestehenden Anspruch auf Einstellung habe. ${ }^{8}$ Das Arbeitsgericht Marburg entschied im Rahmen des $\ 9$ TzBfG, dass der Arbeitnehmer solange einen Anspruch auf Verlängerung der Arbeitszeit habe, solange eine abstrakte Möglichkeit zur Verlängerung bestehe. Auf den konkreten Arbeitsplatz komme es nicht an. 59

\section{Prozessuale Geltendmachung der Ansprüche}

Unterschiede sind in den Niederlanden und in Deutschland auch bei der prozessualen Geltendmachung der Ansprüche auf Anpassung der Arbeitszeit zu beobachten. In den Niederlanden wurden die meisten Klagen im Eilverfahren entschieden. Nur in Ausnahmefällen wurde die Möglichkeit einer Anpassung des Arbeitsvertrags nach dem Waa im Wege des Eilverfahrens generell abgelehnt oder aufgrund der fehlenden Möglichkeit, im Eilverfahren zu überprüfen, ob schwerwiegende betriebliche Belange auf Seiten des Arbeitgebers vorlägen. Die meisten Gerichte wendeten das Eilverfahren ohne Bedenken an. Eine besondere Prüfung der Voraussetzung des Eilverfahrens fand meistens nicht statt. ${ }^{60}$ Auch die vorläufige Vollstreckbarkeit vieler Entscheidungen wurde ohne weitere Diskussion angenommen. ${ }^{61}$

54 Für eine Beschränkung auf die Verringerung: Boewer, TzBfG, Rn. I64.

5s BAG, Urt. v. I 8. 2. 2003-9 AZR I64/02-, NZA 2003, I 392, I 394 f.; bestätigend BAG, Urt. v. I9. 8. 2003-9 AZR 542/02 -, AP TzBfG $\ 8$ Nr. 4; BAG, Urt. v. I6.3.2004 - 9 AZR 323/03 - (Fn. 43), Rn. 79.

56 Ktg. Maastricht, 2. 2. $200 \mathrm{I}$, JAR 200I, 49; Ktg. Nijmegen, I 4. 9. $200 \mathrm{I}$, JAR 2001, 227; ArbG Marburg, Urt. v. I 2. 7. 2002 - 2 Ca 666/oI -, Juris-Nr.: KARE 600006770; BAG, Urt. v. 25 . I0. I994- 3 AZR 987/93-, AuR $200 \mathrm{I}, \mathrm{I} 46 \mathrm{ff}$. zu einer vergleichbaren Tarifregelung.

57 Ktg. Maastricht, 2. 2. $200 \mathrm{I}$, ebd.

58 BAG, Urt. v. 25. I0. I $994-3$ AZR 987/93-, AuR 200I, I46ff.

59 ArbG Marburg, Urt. v. I 2. 7. 2002 - 2 Ca 666/oI -, Juris-Nr.: KARE 600006770.

60 Burri/Opitz/Veldman (Fn. 26), 32 I, 33 If.

6I Siehe: Opitz (Fn. 27), $\mathbb{\$} 3$, B., II., 8. 
In Deutschland wurde hingegen nur in Ausnahmefällen im einstweiligen Rechtsschutz entschieden. Die Klage beim Teilzeitanspruch ist zunächst auf die Abgabe einer Willenserklärung des Arbeitgebers zur Zustimmung zur Verringerung gerichtet. Diese wird erst mit Rechtskraft der Entscheidung vollstreckt ( $\$ 894$ Abs. I ZPO). Daher wurde teilweise angenommen, dass eine einstweilige Verfügung immer eine Vorwegnahme der Hauptsache darstelle und folglich nie möglich sei. ${ }^{62}$ Die herrschende Meinung sieht allerdings im verfassungsrechtlichen Gebot auf effektiven Rechtsschutz die Anwendbarkeit der einstweiligen Verfügung begründet. ${ }^{63}$ Des Weiteren reiche ein Antrag auf vorläufige Beschäftigung zu den angepassten Arbeitszeiten. Die deutschen Arbeitsgerichte hielten unter dieser Voraussetzung die einstweilige Verfügung für möglich, stellten allerdings hohe Anforderungen an den Verfügungsanspruch und den Verfügungsgrund. ${ }^{64}$

Gegen die Entscheidungen zum Waa wurden nur in Ausnahmefällen Rechtsmittel eingelegt. Die meisten Prozessparteien scheinen die Entscheidungen zu akzeptieren. Dies ist hinsichtlich des Bestands des Arbeitsverhältnisses wichtig, da so lange Rechtsstreitigkeiten vermieden werden. In Deutschland wurden hingegen vielfach Rechtsmittel eingelegt. Die wenigsten Entscheidungen wurden akzeptiert, viele Revisionen sind vor dem Bundesarbeitsgericht anhängig, sodass sich in vielen Fällen die Verfahren über Jahre hinziehen. ${ }^{65}$

\section{Rechtsvergleich}

Es lässt sich feststellen, dass ähnliche gesellschaftliche Probleme zu einem ähnlichen Regelinstrument im Arbeitsrecht geführt haben. In Deutschland stand dabei die Bekämpfung der Arbeitslosigkeit im Vordergrund. In beiden Ländern wurden durchsetzbare Ansprüche auf die Verringerung und die Verlängerung der Arbeitszeit geschaffen. Sowohl Art. 2 Waa wie auch $\ 8$ TzBfG kennen eine Einschränkung des Anwendungsgebiets.

Die inhaltliche Schwerpunktsetzung unterscheidet sich allerdings in beiden Gesetzen stark. In den Niederlanden sollte auf der einen Seite der Arbeitnehmer die Möglichkeit erhalten, seine Arbeitszeit flexibel gestalten zu können. Daher wurde ein relativ starkes Recht auf Anpassung der Arbeitszeit geschaffen. Auf der anderen Seite wurde zum Schutz des Arbeitgebers eine relativ schwache Regelung zur Verteilung der Arbeitsstunden gewählt. Hier wurde das Interesse des Arbeitgebers berücksichtigt, den Arbeitnehmer nach seinen Bedürfnissen einzusetzen. Dies kann allerdings kontraproduktive Wirkungen gerade hinsichtlich des Ziels, eine bessere Vereinbarkeit von Beruf und Familie zu erreichen, wenn der Arbeitnehmer aufgrund familiärer Pflichten bei einer Anpassung der Arbeitszeit an eine bestimmte Arbeitszeitverteilung gebunden ist. ${ }^{66}$

In Deutschland liegt der Schwerpunkt hingegen auf dem Teilzeitanspruch. Hier ging es zunächst um das Ziel, den Anteil von Teilzeitbeschäftigten generell zu steigern. Die Möglichkeiten des Arbeitgebers, den Teilzeitantrag abzulehnen, wurden gegenüber

62 Rolfs (Fn. 44), I 29, I 36; für Fälle nach dem Teilzeitanspruch in \ Is Abs. 7 BErzGG: Leßmann, DB 200 I, 94, 99; Schiefer, NZA-RR 2002, 393, 394.

63 Buschmann (Fn. 36), $\$ 8$ TzBfG, Rn. 4 I; Schmidt (Fn. 48), 245, 250; Gotthard, NZA 200 I, I I 83, I I 84 ff.; Dütz, AuR 2003, I6 I ff.

64 Beschreibung bei: Burri/Opitz/Veldman (Fn. 26), 32 I, 338 ff.

65 Opitz (Fn. 27), \$5, C.

66 Burri/Opitz/Veldman (Fn. 26), 32 I, $344 \mathrm{f}$. 
dem niederländischen Gesetz erweitert. Die Verteilung der Arbeitsstunden wurde identisch mit der Verringerung geregelt, sodass mit dem deutschen Gesetz hier ein etwas stärkerer Anspruch als durch das niederländische Gesetz geschaffen wurde. Die Verlängerung der Arbeitszeit wurde hingegen deutlich anders geregelt. Sie fand - trotz der mit ihr verbundenen Einschränkungen für Arbeitgeber - nur wenig Beachtung. Viele Anforderungen, die in $\$ 8 \mathrm{TzBfG}$ enthalten sind, sind hier nicht vorgesehen worden. Die Ablehnungsmöglichkeiten des $\$ 9$ TzBfG sind anders strukturiert. Es fehlt eine Regelung zur Verteilung der Arbeitszeit, sodass das allgemeine Direktionsrecht des Arbeitgebers gilt. ${ }^{67}$ Auch in der Rechtsprechung hat die Verlängerung der Arbeitszeit noch keine Bedeutung erlangt. Dies wird sich vermutlich noch ändern, wenn der Wechsel zwischen verschiedenen Arbeitszeiten im Laufe einer Erwerbsbiographie üblicher und akzeptierter wird.

Die schwerwiegenden betrieblichen Belange wirken in den Niederlanden etwas stärker als die betrieblichen Gründe in Deutschland. In beiden Ländern müssen die Ablehnungsgründe noch eine qualifizierende Voraussetzung erfüllen. In den Niederlanden müssen sie zu ernsthaften Problemen und in Deutschland zu einer wesentlichen Beeinträchtigung des Betriebes führen. Die Behauptung einer unternehmerischen Entscheidung ohne weitere Begründung kann auch in Deutschland keinen betrieblichen Grund, der zur Ablehnung des Teilzeitanspruchs berechtigt, darstellen. Die unternehmerische Freiheit beinhaltet kein Recht, im eigenen Betrieb bestehende Gesetze nicht anzuerkennen. ${ }^{68}$

Bei einer längeren Geltung werden auch die Erfahrungen mit der Elternteilzeit, die bisher aufgrund der Geltungsdauer des BErzGG noch keine Rolle spielten, Bedeutung für die Frage, ob betriebliche Gründe vorliegen, erlangen. ${ }^{69}$

In beiden Ländern haben die meisten Kläger familiäre Verpflichtungen und sind so prinzipiell auf eine schnelle Entscheidung angewiesen. In den Niederlanden hat sich daher das Eilverfahren als Standard durchgesetzt. Dass in Deutschland relativ wenig Gebrauch von der einstweiligen Verfügung gemacht wird, lässt sich auf der einen Seite auf die unklare rechtliche Situation, aber auch auf den längeren Gewährungszeitraum der Elternzeit zurückzuführen. ${ }^{\circ}$

\section{E. Scblussfolgerungen für Deutschland}

Die Einführung der Ansprüche auf Anpassung der Arbeitszeit haben nicht zu der befürchteten »Prozesslawine « geführt..$^{11}$ In Deutschland bestand zwar im Gegensatz zu den Niederlanden das Bedürfnis, die Fragen im Rahmen des Teilzeitanspruchs durch das Bundesarbeitsgericht klären zu lassen. Es ist aber nicht zu einer Flut von Klagen gekommen. Dies wird auch daran liegen, dass die meisten Arbeitnehmer eine einvernehmliche Lösung mit dem Arbeitgeber zu finden versuchen, um ihren Arbeitsplatz nicht zu gefährden..$^{2}$

Das TzBfG wird in Deutschland zu mehr Verbreitung und Akzeptanz von Teilzeitarbeit führen. Ein uneingeschränkter Rechtsanspruch wurde mit dem TzBfG nicht geschaffen, jedoch müssen sich viele Arbeitgeber nun erstmals mit der Frage be-

67 Zwanziger (Fn. 36), \$9 TzBfG, Rn. I 3.

68 LAG Baden-Württemberg, Urt. v. 27.3.2002 - I2 Sa I 24/OI -, Juris-Nr.: KARE 6000065 I 5 .

69 Burri/Opitz/Veldman (Fn. 26), 32 I, 342.

70 Burri/Opitz/Veldman (Fn. 26), 32 I, 345.

7I Burri/Opitz/Veldman (Fn. 26), 321, 343; Wanger (Fn. I9), S. 7.

72 Däubler, ZIP 2000, I96I, I963. 
schäftigen, ob Teilzeitarbeit in ihrem Betrieb möglich ist. Dass durch die Regelung ein Einstellungshemmnis und damit ein Bumerangeffekt für Frauen ${ }^{73}$ geschaffen wurde, lässt sich bisher nicht feststellen. Andere arbeitsrechtliche Regelungen wie der Mutterschutz und die Elternzeit seien größere Barrieren bei der Einstellung.7 ${ }^{74}$ Ein gewisser Schutz vor einem solchen Effekt beim Teilzeitanspruch besteht darin, dass alle Arbeitnehmer unabhängig von Gründen ihn geltend machen können.75 Würde die teilweise geführte Diskussion dazu führen, diesen Anspruch nur aus familiären Gründen zu gewähren, könnte dies einen negativen Beschäftigungseffekt für Frauen zur Folge haben, da insbesondere Frauen Familienarbeit leisten.

Durch die teilweise ideologisch geführte Diskussion wurde der Blick auf andere Probleme verstellt. Die Frage, welche Rolle die Einschränkung hat, die Verteilung der Arbeitsstunden nach Bedürfnissen des Arbeitnehmers festzulegen, und welche Einschränkungen mit dem Recht auf Verlängerung der Arbeitszeit verbunden sind, wurde nur am Rande thematisiert. Für den deutschen Arbeitnehmer hat dies zwar den Vorteil, dass das TzBfG ein stärkeres Recht auf eine bestimmte Verteilung der Arbeitsstunden als das Waa enthält. Allerdings verdeutlicht dies, dass keine sachliche Diskussion über die Frage der Anpassung der Arbeitszeit geführt wurde. Auch in der Rechtsprechung zeigt sich, dass im Vergleich zu den Niederlanden Teilzeitarbeit in Deutschland unüblich ist und noch viele Vorbehalte gegen Teilzeitarbeit bestehen. Nach einer längeren Geltung tritt vermutlich eine gewisse Gewöhnung an das TzBfG ein. Damit kann die Chance für Arbeitnehmer, lediglich durch eine Beratung mit dem Arbeitgeber eine Anpassung der Arbeitszeit zu erreichen, steigen, allerdings können die gerichtlichen Durchsetzungsmöglichkeiten sinken. Die Streitigkeiten werden sich vermutlich weg von der Frage der Verringerung der Arbeitszeit und hin zu der Frage der Verteilung der Stunden entwickeln.

Eine reine Regulierung über den Markt, wie dies teilweise gewünscht und nun auch wieder vom Deutschen Juristentag gefordert wurde, hätte in Deutschland keine positiven Auswirkungen auf die Entwicklung von anderen Arbeitsformen. In der Diskussion um das TzBfG wurde und wird von einem traditionellen Verständnis der Erwerbsarbeit ausgegangen. Die Norm stellt dabei der männliche Vollzeitarbeitnehmer ohne familiäre Pflichten dar. Ohne gesetzliche Regelungen würde der Markt zu spät auf die sich verändernde Gesellschaft reagieren. Denn die deutsche Gesellschaft steht vor der großen Aufgabe, auf der einen Seite aktuell mehr Arbeitsplätze zu schaffen und längerfristig einem Mangel an qualifizierten Arbeitskräften vorzubeugen. Erschwerend kommt ein Rückgang der Geburtenanzahl hinzu, der auch auf die fehlende Entwicklung von neuen Formen der Gestaltung von Erwerbs- und Familienarbeit zurückzuführen ist. ${ }^{76}$

In den Niederlanden wird schon seit längerem über die Frage eines einheitlichen Levensloopbaanbeleid diskutiert77 und erste Schritte zu einer einheitlichen Betrachtung der Lebenserwerbstätigkeit getan. Versucht wird, den Wechsel zwischen verschiedenen Arbeitszeiten zu vereinfachen, um den in unterschiedlichen Lebensphasen notwendigen Wechsel von Zeiten der Teil- und Vollerwerbstätigkeit und so auch die Vereinbarkeit von Familie und Beruf zu ermöglichen. ${ }^{78}$ Es ist zu hoffen, dass auch in Deutschland eine stärkere Diskussion um diese Fragen stattfinden wird. Es sollte

73 Diese Befürchtung äußert Schmidt, in: ILJ 200I, 335, 35 I.

74 Wanger (Fn. 19), S. 5.

75 Ähnlich: Schiek (Fn. 24), S. I 8, 40.

76 Ausführliche Beschreibung bei: Rürup, Nachhaltige Familienpolitik im Interesse einer aktiven Bevölkerungspolitik, 2003 .

77 Siehe hierzu: Burri (Fn. 32), $70 \mathrm{ff}$.

78 de Wolff, Wet arbeid en zorg, 2002. 
geklärt werden, welche Anpassungen der Arbeitsbedingungen im Rahmen einer

Gesamtbetrachtung des Lebenslaufs möglich und notwendig sind, um mit den Folgen einer alternden Gesellschaft umzugehen und gleiche Möglichkeiten für Männer und Frauen und bessere Bedingungen für Kinder zu schaffen.

Die in das TzBfG gesetzten Erwartungen werden durch das Gesetz allein nicht erfüllt werden können. Die befürchteten negativen Folgen, wie etwa ein Nachteil für den Standort Deutschland oder eine Einstellungspraxis, die Frauen wegen des Gesetzes benachteiligt, lassen sich allerdings auch nicht nachweisen. So ist das Gesetz zumindest ein Schritt zu einer Neudefinition der Arbeitsbeziehungen.

\title{
Karsten Brandt/Matthias Mittag Grenzen polizeilichen Handelns
}

\author{
Kriminalpolitische und rechtliche Beurteilung sog. Graffiti- \\ Verordnungen
}

Nach mehreren erfolglosen Gesetzesinitiativen, den Straftatbestand der Sachbeschädigung $\left(\mathbb{3}_{303}\right.$ StGB$)$ um die Variante des »Verunstaltens« fremden Eigentums zu erweitern, haben einige Bundesländer in Eigenregie gehandelt und sog. GraffitiVerordnungen erlassen. Solche Polizeiverordnungen verbieten das Verändern des Erscheinungsbildes fremder Sachen durch Graffiti und sanktionieren ein Zuwiderhandeln als Ordnungswidrigkeit. Der Beitrag geht der kriminalpolitischen Zweckmäßigkeit und der verfassungs- und polizeirechtlichen Zulässigkeit dieses Vorgehens am Beispiel der sächsischen Graffiti-Verordnung nach.

\section{Einleitung}

»Weil der Bund nicht handelt: Sachsen sanktioniert illegale Graffiti«. So titelt die Pressemitteilung des Sächsischen Staatsministeriums des Innern vom 26. 2. $2004^{\mathrm{I}}$ anlässlich des Erlasses der sächsischen Graffiti-Verordnung. ${ }^{2}$ \ I dieser Polizeiverordnung verbietet, »ohne Zustimmung des Eigentümers auf eine Sache durch das Auftragen von Farbe oder anderen Substanzen oder das Abtragen von Material einzuwirken«. $\mathbb{2}$ stuft ein vorsätzliches Zuwiderhandeln als Ordnungswidrigkeit ein und sanktioniert dies mit einer Geldbuße von bis zu Ioo० Euro. Es handelt sich dabei nicht um einen sächsischen Alleingang. Erstmals hat Sachsen-Anhalt ${ }^{3}$ im Jahre 2002 eine landesweite Graffiti-Gefahrenabwehrverordnung erlassen, Hamburg,4

I Pressemitteilung im Internet (eingesehen am 20. I2. 2004), abrufbar unter: http://wosappr.saxony.de/app/ WebObjects/mspublic.woa/wa/componentDetails? $i d=I 0074$.

2 SächsGVBl. 2004, S.65.

3 Graffiti-Gefahrenabwehrverordnung v. I 2. 8. 2002, GVBl. LSA 2002, S. 360 . Die Polizeiverordnung verbietet und bewehrt mit Bußgeld, »unbefugt das Erscheinungsbild einer fremden Sache gegen den Willen der Eigentümerin oder des Eigentümers oder einer sonst berechtigten Person durch die Aufbringung von Farben oder anderen Substanzen oder die Anbringung von Gegenständen nicht lediglich unerheblich zu verändern.«

4 Graffiti-Verordnung v. 2.9. 2003, HmbGVBl. 2003, S. 462. Dort wird verboten und bußgeldbewehrt, »unbefugt das Erscheinungsbild einer fremden Sache gegen den Willen des Berechtigten durch das 